\title{
New satellite sensors for monitoring mining areas: a look at the future
}

\author{
J Morgan TRE ALTAMIRA Inc., Canada \\ A Boudreau TRE ALTAMIRA Inc., Canada \\ MA Verdugo TRE ALTAMIRA S.L., Spain \\ F Meloni TRE ALTAMIRA s.r.l., Italy \\ D Colombo TRE ALTAMIRA s.r.l., Italy
}

\begin{abstract}
Hazard mitigation and risk reduction are becoming increasingly important for mining operations as highlighted by recent catastrophic failures of tailings dams worldwide. These events have focused industry attention on the importance of developing and implementing effective monitoring strategies on all mine sites.

Pushed by the increasing requirements of the mining industry, monitoring services are rapidly evolving to provide tools and methods to identify slope instabilities and manage the associated risks, pointing to the importance of a synergistic use of in situ instruments and remote sensing data. In particular, satellite imagery and InSAR displacement maps are becoming a standard tool to implement a robust and repeatable monitoring program. InSAR has been revealed to be an easy-to-integrate companion of in situ monitoring sensors, such as ground-based radars, Robotic Total Stations and geotechnical instruments, and has proven itself as an effective technology for monitoring block caving induced subsidence and instabilities over large infrastructure such as tailings dams, providing a unique synoptic view which cannot be obtained by means of in situ observations.

Limitations of InSAR are currently related to the frequency of acquisition of satellite sensors, as well as the limited spatial resolution of displacement data when using sensors designed for monitoring large areas. While high spatial resolution $(1 \times 1 \mathrm{~m})$ can be achieved on small areas using commercial sensors, the current sampling frequency of displacement data is at best measured in several days, severely limiting the effectiveness of this tool for feeding early-warning systems.

In this scenario, new constellations of radar sensors are being launched and will shortly become available. This article aims to provide a comprehensive overview of the potential impact on mining monitoring services with a new generation of microsatellites focusing on active radar sensors. Designed to provide high-resolution radar imagery, these data sources will be operational day and night, independent of weather conditions, and allow much more accurate monitoring. With daily updates, InSAR products will see a strong improvement in their ability to detect any changes in displacement trends, allowing operators to develop more effective early-warning systems based on time series analysis. The use of machine-learning and pattern recognition algorithms over hundreds of assets will make it possible to significantly increase the level of knowledge on slope instability, eventually leading to significant risk reduction.
\end{abstract}

Keywords: satellite InSAR, monitoring, TSF monitoring

\section{Introduction}

Implementing a risk management and mitigation program is a necessary way to ensure safe and profitable a mining operation, and the culture of 'safety first' is now common at major mine sites. Predicting the time of failure of slope instabilities and tactical monitoring of surface deformation have become common practice 
thanks to radars and Robotic Total Stations. InSAR recently joined the portfolio of monitoring solutions thanks to its capability of extending the monitoring scale to the entire mine site facilitating strategic monitoring.

The first InSAR deformation maps were produced in the 1990s to measure the effect of an earthquake using satellites acquiring a single acquisition per month. Several academic research groups developed methodologies providing information related to subsidence mapping, landslide activity status and seismic fault identification. InSAR technology evolved rapidly in recent years, in an effort to support a fully remote monitoring service with improved satellite constellations, high-resolution sensors, and the development of automatic, robust and scalable processing algorithms.

A modern InSAR service is built around the idea of displacement updates, provided with every new acquisition, and the exploitation of time series of deformation describing the evolution of surface displacement in terms of velocity, acceleration and seasonality. While the fastest repeat cycle available for a single satellite is in the range of 6 days, it is possible to provide updates every 2 days using a variety of constellations acquiring simultaneously.

Two main factors are changing rapidly the use of InSAR services:

1. High-resolution maps, based on sensors capturing images with a spatial resolution up to $1 \times 1 \mathrm{~m}$ providing accurate maps of the displacement and allowing the identification of small areas of displacement.

2. The capacity to acquire data systematically in two orbit directions (called ascending and descending) allowing the discrimination between vertical and horizontal movement over the entire planet.

InSAR data can also be integrated in most monitoring software platforms providing comprehensive visualisation of monitoring information to the technical team.

This article explores the future of InSAR services by discussing the new generation of SAR constellations being designed, their data products, and their potential on InSAR products and services. In the meantime, cloud computing is providing processing power that was unimaginable just a few years ago, and artificial intelligence is providing new powerful tools for processing data and producing actionable and usable information for the client.

\section{$2 \quad$ InSAR today}

SAR satellites navigates along near-polar orbits (i.e. approximately parallel to the north-south direction) and look at the Earth surface from an inclined position. Like other radar systems, SAR satellites are sensitive to surface change occurring along a line-of-sight (LOS). This feature has two major implications for InSAR analyses: First, basic InSAR measurements are a one-dimensional (1D) measurement along the sensor's LOS of the true vector of deformation. Second, satellite InSAR is not sensitive to possible horizontal north-south components of motion due to the orbit direction (Ferretti 2014).

By combining the Earth's rotation and the satellite orbital paths, the entire surface of the Earth is captured in two different satellite geometries: ascending (or east-looking), when the satellite travels from south to north, and descending (or west-looking), when the satellites travels from north to south. Both geometries are considered independent datasets that can be combined to enhance the information provided from a single geometry. Figure 1 shows the geometric rationale of motion decomposition. 

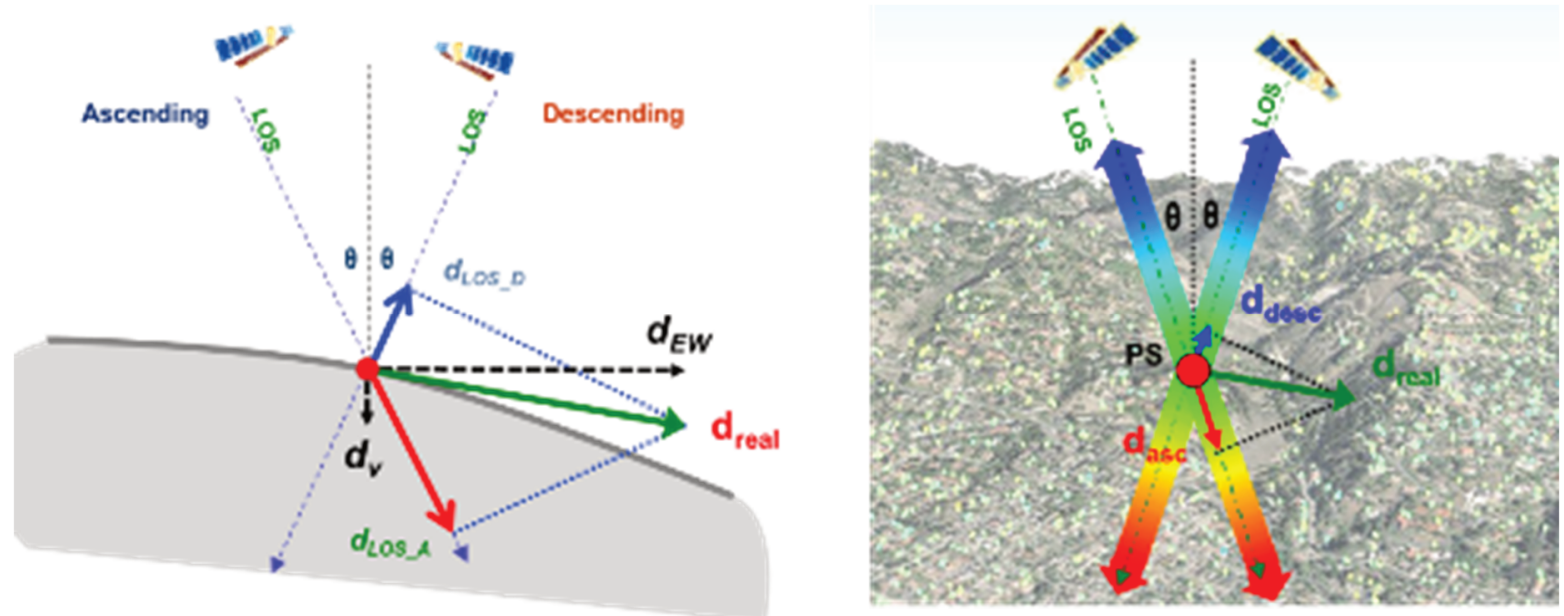

Figure 1 Vertical and horizontal east-west decomposition rationale

Given that SAR satellites look at the ground at an angle, and considering the slope geometry at a site, some areas may not be visible to the satellite's LOS. In areas with strong topography such as an open pit or a tailings dams, the use of a single orbit (ascending or descending) may miss steep portions on one side of the pit. Viewing the area from two orbits allows more information to be captured within the pit. Moreover, Therefore, by referring to a single geometry, the information retrieved by a satellite in unstable areas is whether something is moving away from the satellite or towards it. Conversely, when using two acquisition geometries, it is possible to calculate the real vertical and horizontal (east-west) component of motion. This process simplifies geotechnical interpretation and to graphically plot displacement cross-sections where 2D deformation vectors are overlaid on the topographic profile.

For this reason (Paradella et al. 2015), double geometry is the preferred InSAR monitoring configuration for mine sites as it:

- Maximises the coverage of ground features, especially in areas with strong topography.

- Simplifies the interpretation of InSAR results by providing true vertical and horizontal east-west data instead of simple LOS measurements.

- Enhances the integrability of InSAR data with geotechnical sensors, automatic total stations, levelling, global navigation satellite system and slope stability radars.

\subsection{Sentinel: a game changer, with limitations}

One of the main factors that previously hampered the availability of InSAR as turnkey solution for mine operation monitoring, was a scarcity of single geometry SAR data worldwide, let alone double geometry datasets. In this regard Sentinel-1, a constellation of low-resolution ( $20 \times 4 \mathrm{~m})$ twin satellites operated by ESA, has been a true game changer. Its first satellite Sentinel-1 $A$, was the first SAR satellite to ensure a worldwide acquisition plan with a 12-days revisit cycle, and when Sentinel-1 B operating with the same acquisition frequency joined in October 2016, the newly formed Sentinel-1 constellation started acquiring data in double geometry every six days. Despite its commitment to acquire continuously worldwide, the Sentinel-1 constellation is not ensuring the same performances in all geographical areas. As shown in the (Table 1), less than half the globe is covered in 2D due to a priority in covering the seismologically active 'ring of fire'; nearly two-thirds of the land is only covered in a single geometry. 
Table 1 Sentinel-1 worldwide data coverage

\begin{tabular}{llll}
\hline Area & Area covered in 2D & Mines covered in 2D & Notes \\
\hline North America & $44 \%$ & $31 \%$ & \\
$\begin{array}{l}\text { South and Central } \\
\text { America }\end{array}$ & $32 \%$ & $72 \%$ & $\begin{array}{l}\text { Brazil is not } \\
\text { covered with 2D }\end{array}$ \\
Africa & & & \\
Asia & $21 \%$ & $50 \%$ & \\
Oceania & $51 \%$ & $33 \%$ & \\
Europe & $32 \%$ & $8 \%$ & \\
Total & $100 \%$ & $100 \%$ & \\
\hline
\end{tabular}

When considering mine sites, it is interesting to note that in Central and South America, a large majority of the operations are covered in 2D data with Sentinel-1 and in proximity of active seismic areas.

It is also important to note that Sentinel-1 cannot be tasked for any commercial purpose, as per their specific ESA mandate (https://sentinel.esa.int/web/sentinel/missions/sentinel-1/observation-scenario).

\subsection{The power of high resolution}

As for any remote sensing technique, the spatial resolution of the imagery used in the production of InSAR analyses significantly impacts the capability of the resulting products.

InSAR products typically use the following three classes of resolution:

- Standard resolution $\sim 20 \times 4 \mathrm{~m}$ imagery - pixel size $\sim 80 \mathrm{~m}^{2}$.

- High resolution $\sim 3 \times 3 \mathrm{~m}$ imagery - pixel size $\sim 9 \mathrm{~m}^{2}$.

- Very high resolution $\sim 1 \times 1 \mathrm{~m}$ imagery - pixel size $\sim 1 \mathrm{~m}^{2}$.

The comparison in the capacity to capture the details of an asset is immediately visible in Figure 2 comparing standard resolution to high-resolution data.

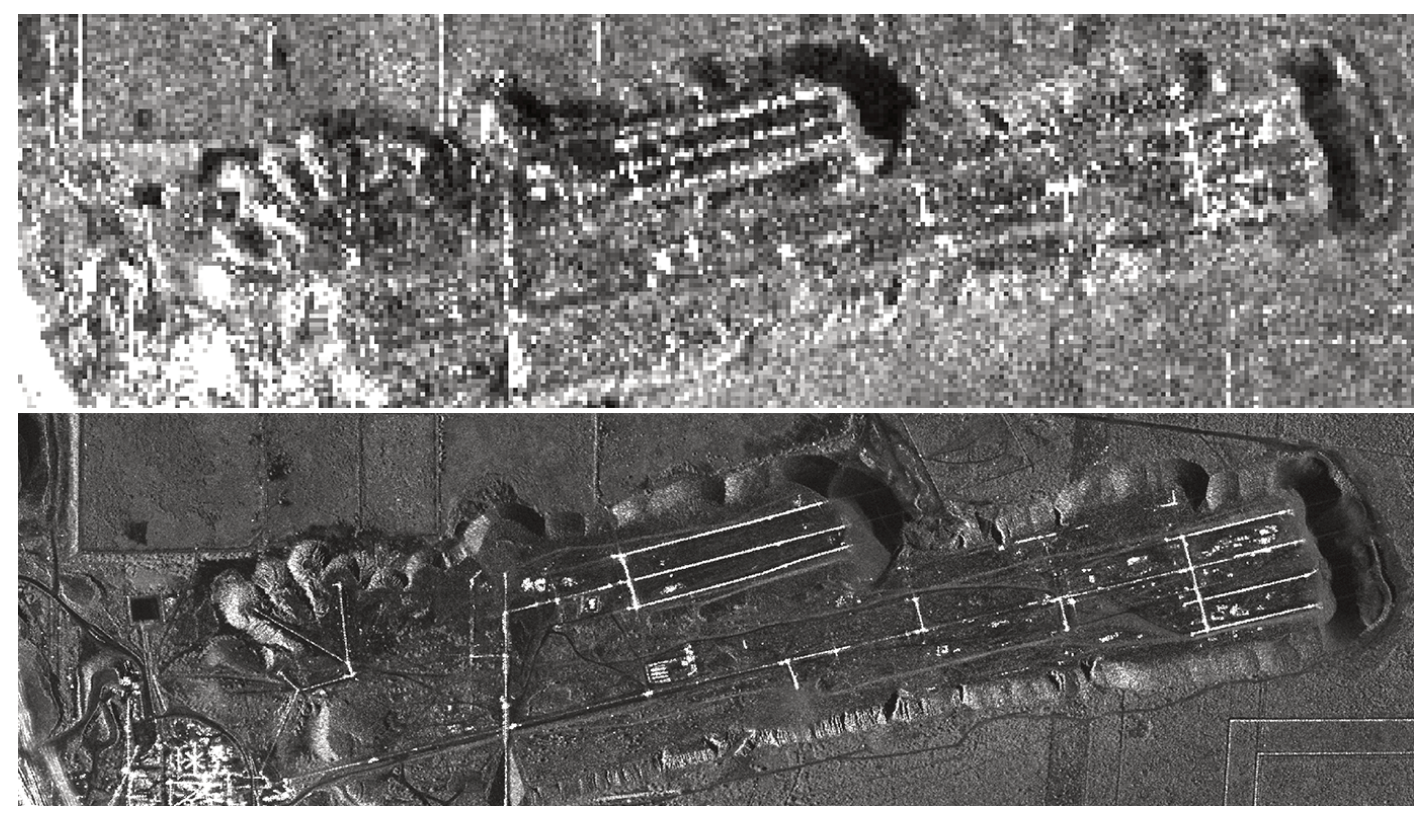

Figure 2 SAR images acquired over the same asset. Sentinel-1 ( 20x4 m) (top); COSMO-SkyMed $(\sim 3 \times 3 \mathrm{~m})$ (bottom) 
While it is possible to achieve millimetric precision in the deformation measurements with any of the three classes of resolution, it is worth mentioning that a higher spatial resolution offers the opportunity to distinguish comparatively smaller areas of significant movement.

Figure 3 is an example showing the relative comparison in the minimal size of a detectable area of movement using standard resolution to the right and high-resolution data to the left.

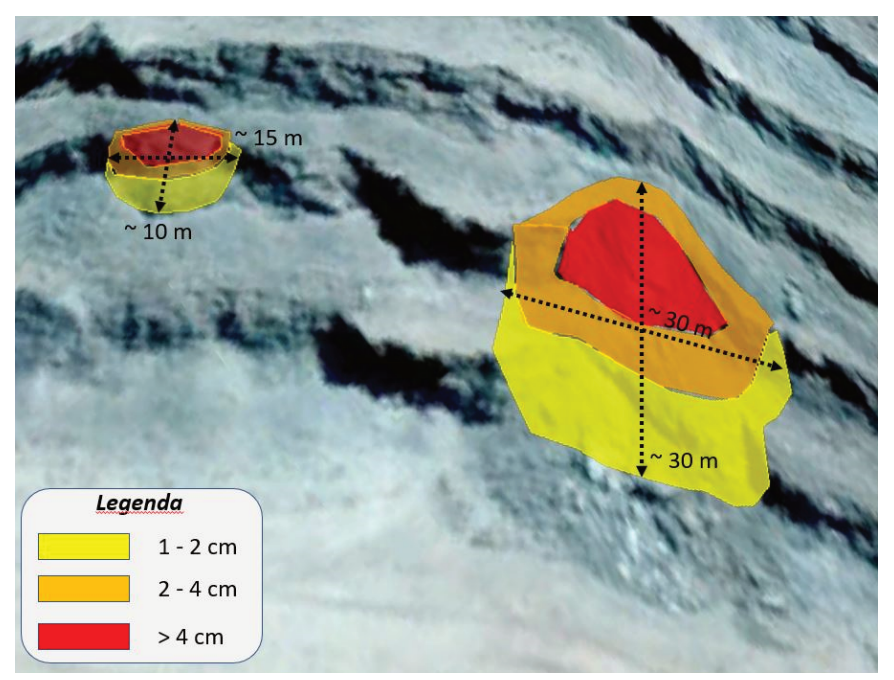

Figure 3 Relative comparison of movement detection capability based on spatial resolution. To the left is the minimal size of displacement that can be detected using high-resolution data, while the right side shows the minimal size of detectable area using standard resolution data

Two slope instabilities are represented. The area on the right spans for almost $1,000 \mathrm{~m}^{2}$, while the smaller area in on the left is in the range of nearly $150 \mathrm{~m}^{2}$.

Figures 4 and 5 show the respective capabilities of standard and high-resolution satellites to detect the deformation occurring on the benches. The sampling grid overlaying the image represents the respective spatial resolution of the sensors (or 'pixel size').

Low-resolution data is able to distinguish significant patterns of deformation where the displacement area is approximately the size of a hectare of land $\left(10,000 \mathrm{~m}^{2}\right)$. In smaller areas, the large pixel size provides coarse delineation leading to an underestimation of actual displacement.

It is worth noting that this theoretical exercise does not consider any noise contribution; when applying the same logic to a 'real world' example the usability of standard resolution data can be further dampened.

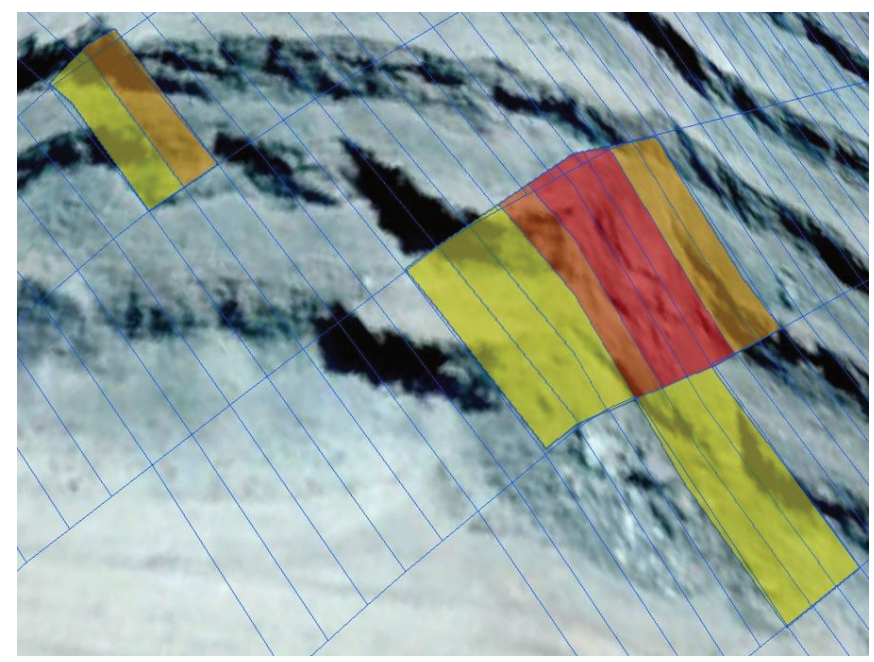

Figure 4 Standard resolution satellite, areal detection capacity 


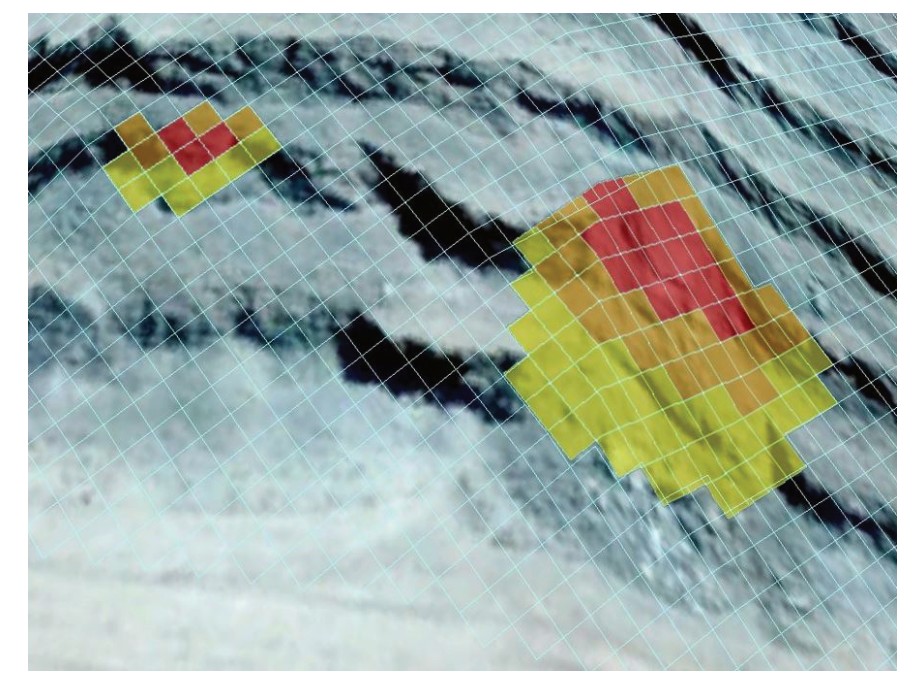

Figure 5 High-resolution satellite, improved areal detection capacity

\section{Satellite radar sensors: a clear trend}

For any monitoring technology, a key element is the number of available data sources. Starting a monitoring program relying on a single satellite can be risky. In fact, a failure of the sensor, downlink, or just a damage to the antenna or solar panel from collision with space debris (a topic that is becoming a real issue in any space program) can compromise the mission and the monitoring program.

Compared to 1991, when just one SAR sensor was available for civilian applications (the ESA ERS-1 mission), today we have more than 15 options, operating at different frequencies and offering different spatial resolutions, orbits and revisit times. Normally, data gathered from different sensors and different acquisition geometries cannot be merged to generate SAR interferograms; however, redundancy results in a more robust monitoring program and would allow the flexibility to switch from one data source to another, should it become necessary.

The number of SAR data sources is increasing steadily, and not only due to space agencies. The growing demand for earth observation (EO) data, together with the reduced cost of satellite sensors and launches, is paving the way for the private sector to join. There is a strong demand for EO data unsusceptible to daylight or cloud coverage and it is becoming evident that radar data is able to compliment optical images in satellite monitoring solution.

To an extent, the SAR business is closely following the same path as optical remote sensing: from extremely expensive missions providing data primarily for intelligence and security purposes and paid for by governmental agencies, to constellations of small, relatively inexpensive microsatellites providing data to an ever-increasing number of commercial applications. It is now possible to manufacture and launch a satellite mounted with SAR sensors at only a fraction of the cost associated with this kind of mission 15 years ago. Business plans involving SAR satellites are gaining increasing interest from investors despite a high-risk factor still associated with the aerospace sector.

The trend is clear: The near future will see several constellations of EO satellites operated by private companies. Table 2 reports a list of six companies targeting the EO market and specifically aiming at operating constellations of SAR sensors. 
Table 2 Main providers related to the business of small SAR sensors

\begin{tabular}{llll}
\hline Company & Country & Constellation target size & Notes \\
\hline ICEYE & Finland & 18 (4 already in orbit) & www.iceye.com \\
CAPELLA Space & USA & 36 & www.capellaspace.com \\
Umbra Lab & USA & 12 & www.umbralab.com \\
SYNSPECTIVE & Japan & 25 & www.synspective.com \\
Urthecast: OptiSAR & Canada & 8 & www.urthecast.com/ \\
XpresSSAR & USA & 8 & www.xpresssar.com \\
\hline
\end{tabular}

Almost all designs share the following similarities:

1. Data redundancy: Rather than using a single sensor, these new constellations will have many smaller and less expensive sensors instead of the large expensive single sensors operated by space agencies. Thus, the potential failure of one sensor will not compromise the monitoring capability of the constellation. Satellite sensors will easily be added, replaced or upgraded as needed.

2. High-resolution and short revisit cycles: Most new SAR satellite operators are designing sensors with high spatial resolution $(<1 \mathrm{~m})$ and very short revisiting time. Daily acquisitions will become standard with the possibility to increase the frequency up to 3-4 images/day. This will be a significant improvement for InSAR monitoring programs, making them much more effective overall. Sub-meter resolution will allow the installation of small, inexpensive, passive, artificial reflectors in areas covered by vegetation and snow.

3. X-band: Most companies are designing and manufacturing sensors operating in X-band. This frequency band allows designers to reduce the antenna dimensions and increase the bandwidth of the system ultimately improving the spatial resolution of the images. Moreover, X-band data maximises the sensitivity to displacement providing better accuracy for InSAR monitoring programs.

4. Limited spatial coverage: Rather than providing a global continuous coverage such as with Sentinel-1, the new generation of SAR sensors will be designed to monitor individual assets anywhere in the world. Typically, high-resolution SAR images cover areas limited to a few hundred square kilometres, although with very short revisiting times.

5. Fast delivery: The market is asking for high-resolution data and timely delivery. All new constellations aim to reduce delivery times from a few days to a couple of hours maximum. This feature will have a strong impact on InSAR monitoring programs, making systems more effective at providing early warnings.

Based on the present scenario and the information available today, the next few years will see space agencies and governments continue to finance powerful (and expensive) sensors capable of acquiring data all over the planet with a resolution of a few meters and a temporal frequency of a few days. Small and relatively inexpensive satellites, most likely operating at X-band, characterised by high spatial resolution and short revisit cycles ( $<1$ days) will be operated primarily by private companies. The data will be used-apart from intelligence and security-for applications related to monitoring individual assets (e.g. mining areas, oil and gas reservoirs, etc.) and infrastructures (e.g. pipelines, lifelines, etc.). The more expensive machines will be used for wide area mapping, monitoring natural hazards, climate change-related phenomena, urban expansion, as well as for multiple earth science applications.

The outlook of this new 'golden age' of SAR sensors appears very promising but there are challenges. Making a small and inexpensive satellite SAR sensor is not an easy task to perform. The challenge lies not in the sensors' generation of amplitude images, but in the orbital stability. In fact, a few months ago ICEYE announced the commercialisation of SAR imagery acquired with their own small sensors; however, InSAR applications require 
minimal orbital tubes, stable orbits, high platform stability, and high quality phase measurements (high antenna gains): this is far more challenging for platforms $<100 \mathrm{~kg}$ proposed by most companies.

Undoubtedly, 2020 will be key as at least eight new private SAR sensors are expected to be launched. By 2021, we expect the daily revisiting time to become reality, making a big change in any InSAR monitoring program.

\section{$4 \quad$ Monitoring with InSAR: a future perspective}

A few things will make a strong in impact on the future of InSAR monitoring capabilities:

- Multiple new satellite constellations be launched and operated for commercial applications.

- Continuous improvements in cloud computing capabilities.

- Evolution of machine-learning methods to derive actionable information from big datasets.

Section 3 discussed how, thanks to the growing demand of the industry, the aerospace sector and downstream services are changing from an academic research and development domain to commercial applications and pursuits. High-resolution satellite data is becoming a standard feature for an increasing number of monitoring programs, and the availability of multiple satellite constellations will provide redundancy and robust acquisition plans to support these programs.

Today, InSAR providers can provide updated results every week (or every two weeks) using single satellites, or potentially every 2-3 days using a combination of constellations (i.e. using different sensors tasked over the same area or acquiring data with the same sensor, but with different acquisition geometries); although this solution is typically very expensive and far from optimal.

Over the next few years, new constellations of SAR microsatellites will make it possible to provide final users high-resolution data updated daily (or more frequently), likely becoming the new monitoring standard for mining operation worldwide.

Furthermore, it is expected that advancements in cloud computing will continue to increase processing times by orders of magnitude compared to today. What is now produced in a few days, will be performed in a few hours thanks to an increased level of automation and computational capabilities. The InSAR industry is already implementing machine-learning algorithms and artificial intelligence to run automatic quality checks. The present goal is to fully exploit high-resolution data and create a new generation of signal processing algorithms to maximise data coverage in the pits and provide timely information for geotechnical teams.

The trends discussed in this paper indicate a significantly large generation of data, which will ultimately require to be 'digested' by integration and visualization platforms. This is another challenge faced by InSAR service providers requiring considerable investment and is a very important part of any satellite downstream service. Data clustering and data screening algorithms are already being used to provide effective tools to extract information from this data glut. The implementation of these new algorithms and techniques is already filling the gap between InSAR monitoring to actionable decision-making systems (Raspini et al. 2018; Carlà et al 2019; Figure 6). 

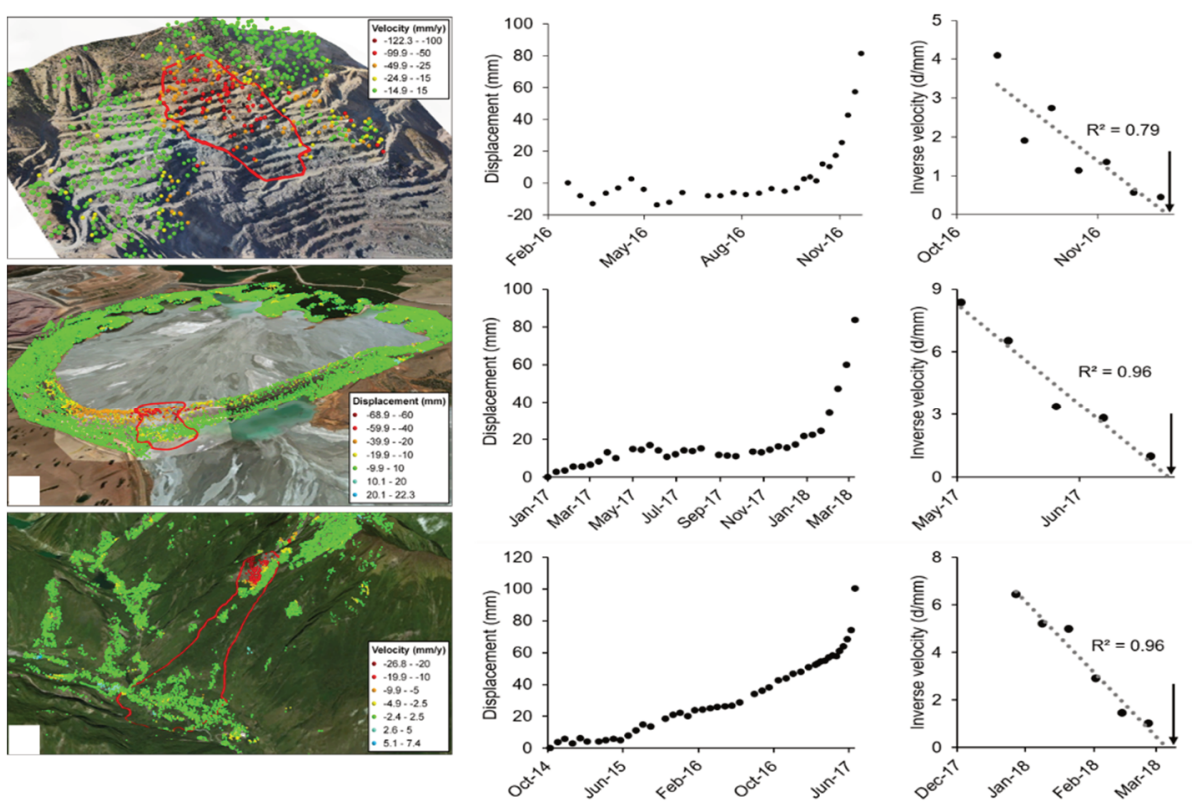

Figure 6 InSAR displacement time series used for failure prediction over an open pit, tailings storage facility embankment and natural landslide (Carlà et al. 2019)

Machine-learning algorithms are already creating a considerable change in many early-warning and decision support systems. Effective monitoring will require effective data screening tools able to identify significant movement and trends amongst thousands of displacement time series updated every day or even every few hours. The tools currently used for in situ instruments (e.g. ground-based radar systems) will also have to be improved and applicable to satellite data. Higher temporal frequencies of observations are expected to make InSAR monitoring programs much more effective and, together with machine-learning algorithms, will allow operators to take advantage of present and past data to predict the future.

\section{Conclusion: InSAR as a service}

In a global monitoring framework, InSAR found its space with Robotic Total Stations, radars and other geotechnical instrumentation to provide actionable information over some of the mine assets. New satellites and microsatellites, and the growing implementation of modern processing methods, will soon allow satellite mapping and measuring to be provided (potentially to every mine in the world) as a daily, routine service.

\section{Acknowledgement}

Authors would like to thank the whole TRE ALTAMIRA staff for all internal discussions and brainstorms about the topic of this paper, as well as for the processing of hundreds of satellite data-stacks acquired by a variety of different satellite sensors.

\section{References}

Carlà, T, Intrieri, E, Raspini, F, Bardi, F, Farina, P, Ferretti, A, Colombo, D, Novali, F \& Casagli, N 2019, 'Perspectives on the prediction of catastrophic slope failures from satellite InSAR', Scientific Reports, vol. 9, issue 1, https://doi.org/10.1038/s41598-01950792-y

Ferretti, A 2014, Satellite InSAR Data-Reservoir Monitoring from Space, EAGE, Houten.

Paradella, WR, Ferretti, A, Mura, JC, Colombo, D, Gama, FF, Tamburini, A, ... \& Gomes, LL 2015, 'Mapping surface deformation in open pit iron mines of Carajás Province (Amazon Region) using an integrated SAR analysis', Engineering Geology, vol. 193, pp. 61-78, doi: 10.1016/j.enggeo.2015.04.015

Raspini, F, Bianchini, S, Ciampalini, A, Del Soldato, M, Solari, L, Novali, F, ... \& Casagli, N 2018, 'Continuous, semi-automatic monitoring of ground deformation using Sentinel-1 satellites', Scientific Reports, vol. 8, issue 1, https://doi.org/10.1038/s41598-01825369-w 
